\title{
Comparison Between Vacuum Sublimed Matrices and Conventional Dried Droplet Preparation in MALDI-TOF Mass Spectrometry
}

\author{
Thorsten W. Jaskolla, ${ }^{\text {a }}$ Michael Karas, ${ }^{\text {a }}$ Udo Roth, ${ }^{\mathrm{b}}$ Kerstin Steinert, ${ }^{\mathrm{b}}$ \\ Christoph Menzel, ${ }^{\mathrm{b}}$ and Karsten Reihs ${ }^{\mathrm{c}}$ \\ ${ }^{a}$ Cluster of Excellence Macromolecular Complexes, Institute of Pharmaceutical Chemistry, Goethe \\ University, Frankfurt, Germany \\ ${ }^{\mathrm{b}}$ Qiagen $\mathrm{GmbH}$, Hilden, Germany \\ ${ }^{c}$ AMF GmbH, Köln, Germany
}

The properties of several cinnamic acid compounds used as matrices for matrix-assisted laser desorption ionization time-of-flight mass spectrometry (MALDI-TOF MS) were investigated as standard dried droplet (DD) and vacuum sublimed preparations. The differences between both preparation methods were analyzed with regard to matrix grain size, internal ion energy, initial velocity, analyte intensity, and analyte incorporation depth. Some of the used cinnamic acid derivatives exhibit clearly reduced grain sizes as sublimed preparations compared with standard DD approaches. In these cases higher effective temperatures could be measured accompanied by increased analyte intensities, which can be explained by stronger volatilization processes caused by a hindered heat dissipation resulting in a raised analyte transfer into the gas phase. For all sublimed compounds, a strong increase of the initial ion velocity compared with DD preparations could be measured. Higher initial ion velocities correlate with a decrease in internal ion energy which might be attributed to the very uniform crystal morphology exhibited by sublimed compounds. For sublimed matrices without reduced grain size, at least slightly higher analyte intensities could be detected at raised laser fluences. Analyte accumulation in the uppermost matrix layers or the detected higher ion stability can be explanations for these results. (J Am Soc Mass Spectrom 2009, 20, 1104-1114) (c) 2009 American Society for Mass Spectrometry

$\mathrm{M}$ atrix-assisted laser desorption ionization mass spectrometry (MALDI-MS) [1-3] has evolved into an essential tool for chemical analysis of biomolecules. Ever increasing numbers of samples call for automation in high-throughput workflows. One of the key areas for progress in automated MALDI analyses consists of improved sample preparation techniques. Numerous efforts have been conducted for improving the preparation step, e.g., the generation of thin uniform matrix layers with high shot-to-shot reproducibility using the aerospray technique by combination of matrix and nitrocellulose [4] or for analysis of synthetic polymers [5]. Also, electrospray deposition and vacuum deposition were reported to yield homogeneous sample preparations with increased analyte intensities [6-9]. Matrix deposition by sublimation for MALDI imaging has been reported to yield more intense signals with less alkali adducts compared with the electrospray technique [10]. Additionally, sublimed matrix spots deposited on extremely hydrophobic surfaces described in [11-14] offer a variety of beneficial prop-

Address reprint requests to Graduate engineer T. Jaskolla, Institute of Pharmaceutical Chemistry, Max-von-Laue-Str. 9, Goethe University Frankfurt, D-60438 Frankfurt, Germany. E-mail: Jaskolla@iachem.de erties compared with standard dried droplet (DD) preparations: The main advantage of sublimed matrix spots compared with standard DD preparations are higher analyte sensitivities [14-17]. This is likely related to the formation of considerably smaller crystals and narrow size distribution of thin-film sublimed matrices, such as $\alpha$-cyano-4-hydroxycinnamic acid (CHCA), compared with DD preparations with comparatively large particle sizes and broader size distribution [15]. As a consequence of this, the specific matrix surface area as well as the amount of potential binding places increases compared with standard DD precipitates, which will result in increased analyte binding properties. Additionally, the process of matrix preparation and spotting is omitted, resulting in reduced sample preparation work, which seems beneficial especially for highthroughput analysis. Moreover, the sublimation process purifies the matrix compound leading to matrix layers of improved purity that homogeneously cover the entire sample spot area. It also has been shown that small and evenly distributed crystals exhibit higher spot-tospot reproducibility compared with standard DD preparations [18], with only partial crystal coverage of the spot surface necessitating the selection of individual crystals or random walking over the spot. Therefore, 
additional time and laser shots are required due to analysis of noncovered or partially covered spot areas. In combination with other features like precisely defined spot size, shape, and location, this approach enables more homogeneous sample preparation conditions and higher reproducibility [14]. Nevertheless, the influence of sublimation on matrix properties is not yet fully understood. Therefore, physicochemical parameters that seem important for the ablation process are compared in detail in this work for standard DD and sublimed matrix preparations for the most widely used matrix CHCA and structurally similar derivatives, which are suitable matrix compounds.

\section{Experimental}

\section{Materials and Reagents}

All chemicals were purchased from Sigma-Aldrich (Taufkirchen, Germany) and were of analytical grade, except p-tolualdehyde, sinapinic acid (SA), and trifluoroacetic acid (TFA), which were of the highest grade available. The in-solution bovine serum albumin (BSA) digest (Starter Kit for MALDI-TOF MS, Part-No.: 208241, Lot: 2004-208241-001) and CHCA (Part-No.: 201344, Lot: 2007-201344-001) were purchased from Bruker Daltonics (Bremen, Germany). Vanillin (99\%) was obtained from Acros (Nidderau, Germany). Acetonitrile (ACN) and methanol $(\mathrm{MeOH})$ were both Rotisolv HPLC gradient grade and purchased from Roth (Karlsruhe, Germany). Water was purified by an ion-exchange system (Milli-Q, Millipore (Schwalbach, Germany). The Sequazyme Mass Standards Kit (calibration mixture 1+2) was obtained from Applied Biosystems (Darmstadt, Germany). Quaternary p-methoxybenzylpyridinium chloride was synthesized by the corresponding benzyl chloride and pyridine as described previously [19]. GELoader Tips were ordered from Eppendorf (Wesseling-Berzdorf, Germany). Sample holders (Mass Spec Turbo Chip Holders) were courtesies from Qiagen N.V. (Hilden, Germany).

\section{Synthesis of CHCA Derivatives}

$\alpha$-Cyano-4-methylcinnamic acid (4-Me-CCA), $\alpha$-cyano4-hydroxy-3-methylcinnamic acid (4-HO-3-Me-CCA), and $\alpha$-cyano-4-hydroxy-3-methoxycinnamic acid (4HO-3-MeO-CCA) were synthesized by standard Knoevenagel condensation reactions using cyanoacetic acid and substituted benzaldehydes. Ammonium acetate was used as catalyst. In a typical approach, 2 g cyanoacetic acid (1 eq), 0.9 eq of the benzaldehyde and $0.15 \mathrm{eq}$ of ammonium acetate were refluxed with stirring in toluene. After quantitative separation of the byproduct water by a water separator the reaction mixture was cooled and filtered. The crude product was washed with sufficient amounts of distilled water and purified by repeated recrystallization from $\mathrm{MeOH} /$ water and vacuum sublimation, if used as sublimed matrices. Overall yields were between $70 \%$ and $90 \%$. 4-Me-CCA:
${ }^{1} \mathrm{H}$ NMR (300 MHz, DMSO-d 6 ) $\delta 2.39(\mathrm{~s}, 3 \mathrm{H}), 7.39(\mathrm{~d}$, $2 \mathrm{H}, \mathrm{J}=6.4 \mathrm{~Hz}), 7.95(\mathrm{~d}, 2 \mathrm{H}, \mathrm{J}=9.0 \mathrm{~Hz}), 8.28(\mathrm{~s}, 1 \mathrm{H}), 13.9$ $(\mathrm{s}, \mathrm{br}, 1 \mathrm{H})$. Accurate mass determination using (CHCA + $\mathrm{H})^{+}$and $\left(\mathrm{CHCA}+\mathrm{H}-\mathrm{H}_{2} \mathrm{O}\right)^{+}$as internal calibrants: $(\mathrm{M}+\mathrm{H})^{+} 188.0708 \pm 0.0001 \mathrm{Da}$ (error: $\left.1.0 \pm 0.5 \mathrm{ppm}\right)$, calc. 188.07061 Da. 4-HO-3-Me-CCA: ${ }^{1} \mathrm{H}$ NMR (300 $\left.\mathrm{MHz}, \mathrm{DMSO}-\mathrm{d}_{6}\right) \delta 2.14(\mathrm{~s}, 3 \mathrm{H}), 6.94(\mathrm{~d}, 1 \mathrm{H}, \mathrm{J}=8.3 \mathrm{~Hz})$, 7.69 (s, 1H), $7.81(\mathrm{~d}, 1 \mathrm{H}, \mathrm{J}=14.0 \mathrm{~Hz}), 8.12$ (s, 1H), 10.69 (s, br, 1H), 13.57 (s, br, 1H). Accurate mass determination using $(\mathrm{CHCA}+\mathrm{H})^{+}$and $(\mathrm{CHCA}+\mathrm{Na})^{+}$as internal calibrants: $(\mathrm{M}+\mathrm{H})^{+} 204.0660 \pm 0.0002 \mathrm{Da}$ (error: $2.4 \pm 1.2$ ppm), calc. $204.06552 \mathrm{Da} .4-\mathrm{HO}-3-\mathrm{MeO}-$ CCA: ${ }^{1} \mathrm{H}$ NMR (300 MHz, DMSO-d $\left.{ }_{6}\right) \delta 3.81(\mathrm{~s}, 3 \mathrm{H}), 6.94$ $(\mathrm{d}, 1 \mathrm{H}, \mathrm{J}=8.3 \mathrm{~Hz}), 7.58(\mathrm{~d}, 1 \mathrm{H}, \mathrm{J}=10.4), 7.74(\mathrm{~s}, 1 \mathrm{H})$, 8.18 (s, 1H), 10.44 (s, br, 1H), 13.60 (s, br, 1H). Accurate mass determination using protonated and sodiated 4-chloro- $\alpha$-cyanocinnamic acid [20] as internal calibrants: $(\mathrm{M}+\mathrm{H})^{+} 220.0598 \pm 0.0003 \mathrm{Da}$ (error: $-2.7 \pm 1.5$ ppm), calc. 220.06043 Da. All NMR chemical shifts correspond to an $\mathrm{AA}^{\prime} \mathrm{XX^{ \prime }}$ spin system.

\section{Thermogravimetric Analysis (TGA)}

The maximum sublimation process temperatures at which only negligible matrix decomposition occur $(\mathrm{dm} / \mathrm{dT}<-0.025 \% / \mathrm{K})$ were detected by TGA and are as follows: $410 \mathrm{~K}$ for $\mathrm{CHCA}, 450 \mathrm{~K}$ for SA, $433 \mathrm{~K}$ for 4-Me-CCA, $443 \mathrm{~K}$ for 4-HO-3-MeO-CCA, and $453 \mathrm{~K}$ for 4-HO-3-Me-CCA (Supplementary Information (SI), which can be found in the electronic version of this article, Figure 1).

\section{Sample Preparation and Measurement}

The peptides of the combined calibration mixtures 1 and 2 were dissolved in $20 \%$ ACN $/ 0.01 \%$ TFA to final concentrations of $125 \mathrm{fmol} / \mu \mathrm{L}$ des-arg ${ }^{1}$-bradykinin, $413 \mathrm{fmol} / \mu \mathrm{L}$ angiotensin I, $163 \mathrm{fmol} / \mu \mathrm{L}$ glu ${ }^{1}$-fibrinopeptide $\mathrm{B}, 6$ $\mathrm{fmol} / \mu \mathrm{L}$ neurotensin, $250 \mathrm{fmol} / \mu \mathrm{L} \mathrm{ACTH} \mathrm{(1-17),} 188$ $\mathrm{fmol} / \mu \mathrm{L} \mathrm{ACTH} \mathrm{(18-39),} 375 \mathrm{fmol} / \mu \mathrm{L} \mathrm{ACTH} \mathrm{(7-38),}$ and $438 \mathrm{fmol} / \mu \mathrm{L}$ bovine insulin. The in-solution BSA digest was dissolved in $30 \% \mathrm{ACN} / 0.1 \%$ TFA to a final concentration of $1 \mathrm{fmol} / \mu \mathrm{L}$. p-Methoxybenzylpyridinium chloride, which was used for the measurements of the effective temperatures, was dissolved in $20 \%$ ACN to final concentrations of 2 and $4 \mathrm{pmol} / \mu \mathrm{L}$. The initial velocities were measured from $350 \mathrm{fmol}$ bovine insulin as analyte $(\mathrm{c}=700 \mathrm{fmol} / \mu \mathrm{L}, \mathrm{V}=0.5 \mu \mathrm{L})$ dissolved in $20 \%$ ACN $/ 0.01 \%$ TFA; $0.5 \mu \mathrm{L}$ matrix solution $(30 \%$ $\mathrm{ACN} / 0.1 \%$ TFA, c $=45 \mathrm{mM}$ for SA; $70 \% \mathrm{ACN} / 0.1 \%$ TFA, $\mathrm{c}=30 \mathrm{mM}$ for all other matrices) and $0.5 \mu \mathrm{L}$ analyte solution were mixed directly on a polished steel target and air-dried for standard DD preparation. In the case of sublimed spots the matrices CHCA, SA, 4-MeCCA, 4-HO-3-Me-CCA, and 4-HO-3-MeO-CCA were deposited by thermal sublimation in a vacuum chamber with a background pressure of about $10^{-5}$ mbar through contact masks with $600 \mu \mathrm{m}$ diameter holes. For focusing effects with reduced spot sizes [21] the gold- 
sputtered aluminum oxide template surfaces were hydrophobically coated before matrix sublimation. The deposited layer thickness per spot was about $3 \mu \mathrm{m}$. Sample preparation on sublimed spots was carried out as surface preparations with $0.5 \mu \mathrm{L}$ analyte solution using Eppendorf GELoader Tips. The sublimed matrix crystals dissolve only partially upon sample deposition, when less than about $50 \%$ (vol/vol) ACN is used. The remaining solid material supposedly acts as seed crystals causing unaltered crystal morphology upon recrystallization.

The spots were washed after air drying by dipping the template into a $20 \mathrm{mM} \mathrm{NH} \mathrm{H}_{4} \mathrm{H}_{2} \mathrm{PO}_{4}$ solution for $3 \mathrm{~s}$. Analyte peak intensities, areas, and signal-to-noise $(\mathrm{S} / \mathrm{N})$ ratios for DD and sublimed spots were detected by measuring four spots/fluence each irradiated with 50 shots $\times 30$ subspectra. No visual changes in the spot shape could be detected after laser penetration indicating that the chosen matrix thickness was sufficiently high (SI Figure 2).

\section{Determination of the Laser Penetration Depth}

For accurate analysis of the laser penetration depth, the depth profile was analyzed by means of a 3D profilometer with atomic force microscope sensor (MicroProf; FRT, Bergisch Gladbach, Germany). For this purpose, sublimed and partially redissolved CHCA layers of higher thickness were used. After 200, 300, and 400 shots, each at the same location with a $355 \mathrm{~nm}$ laser (ABI 4700) adjusted to optimum analyte $\mathrm{S} / \mathrm{N}$ ratios, the crater depth was determined as the difference between the average height of the circumjacent surface and the crater bottom. The overall ablation was determined to $0.9 \mu \mathrm{m}$ in the case of 200 shots, to $1.6 \mu \mathrm{m}$ for 300 shots, and to $3 \mu \mathrm{m}$ for 400 shots (SI Figure 3), which results in an averaged ablation of $6.4 \pm 1.5 \mathrm{~nm}$ per shot. Therefore, nearly 500 shots at the same spot location would be necessary for complete ablation of the sublimed matrix, a value not in the least attained for the PMF approaches and uniform spot irradiation.

\section{Mass Spectrometry}

The initial velocities of the matrix compounds were determined using a Voyager-DE PRO Workstation (Applied Biosystems) in linear ion mode. Accurate mass determination of the synthesized matrices was determined with a Voyager-DE STR Workstation (Applied Biosystems). All other MS measurements were performed with a 4800 MALDI TOF/TOF Analyzer (Applied Biosystems). Calibration of the absolute laser fluence in the 4800 MALDI TOF/TOF analyzer was performed by a mirror in the beam path to deflect the laser beam onto a calibrated fluence probe in a calibrated setup of the deflection mirror (SI Data 1, SI Table 1, and SI Figure 4,5). Careful spot alignment was performed before MS measurements after changing sample plates. For automatic measurements the spot diameter of the sublimed spots was specified to $500 \mu \mathrm{m}$ in the 4800 setup, thus differing from the true value of $600 \mu \mathrm{m}$ to avoid access of the laser beam beyond the matrix coated spot area. For all spectra acquired with the 4800 MALDI TOF/TOF Analyzer the positive ion reflector mode with random and uniform search pattern was used.

\section{Analysis of the Analyte Incorporation Depth Profile}

For obtaining DD and sublimed preparations with identical matrix-to-analyte ratios unused sublimed spots were completely dissolved with $0.5 \mu \mathrm{L} 70 \% \mathrm{ACN}$, thus leading to DD preparations. Such DD spots still exhibit the original spot size diameter because of the hydrophobic spot surrounding. For both preparations $0.5 \mu \mathrm{L}$ of the diluted calibration mixtures 1 and 2 were applied, in the case of DD preparations directly after complete dissolving of the matrix crystals. The analyte incorporation depth was determined using two slightly different approaches. Six independent experiments were performed for DD and sublimed preparations in each approach. First, the surface of each preparation was irradiated 21 times with 20 shots at a constant spot position for "drilling" on single locations. For this purpose, the detector gain was increased to yield sufficiently strong signals because of the low number of shots per spectrum. Second, the surface of each preparation was irradiated 36 times with 500 shots each. During each irradiation cycle 25 subspectra at randomly chosen positions with 20 shots per subspectrum were accumulated to obtain a homogeneous layer-by-layer ablation for the complete spot surface. The same optimized laser fluence was used for both preparation techniques.

\section{Scanning Electron Microscopy}

Scanning electron microscopy (SEM) was performed by nanoAnalytics $\mathrm{GmbH}$ (Münster, Germany) using a LEO 1530 VP Gemini instrument. The spots were imaged after sample preparation as described above without prior MALDI analysis. Before SEM analysis all samples were sputter coated with a $\sim 3 \mathrm{~nm}$ thick platinum film.

\section{Measurement of the Initial Ion Velocity}

Identical hydrophobic coated aluminum oxide templates were used both for DD and sublimed spots. For improved electrical conductivity thus avoiding electrical charging upon laser irradiation a gold coated commercial Mass Spec Turbo Chip Holder from Qiagen was used for loading the templates into the Voyager-DE PRO mass spectrometer. The accordant Mass Spec Turbo Chip Plate File available at www.qiagen.com/ maldiprep was used for sublimed spots. DD spots were traced by photoluminescence upon laser irradiation. 
Device parameters were set to identical values as given in [22]. The delayed extraction (DE) time was varied from $10 \mathrm{~ns}$ (shortest selectable interval) to $1000 \mathrm{~ns}$ in 200 ns steps. To account for the internal delay between trigger and the photo diode signal $200 \pm 20$ ns were added to the DE time. For each DE time the average flight time of the protonated analyte ions was determined from six individual measurements with 50 shots each at $50 \%$ peak centroid after smoothing the spectra using the method of Savitzky-Golay at 251 data points.

\section{Measurement of the Effective Plume Temperature}

Determination of the laser fluence dependent effective plume temperature was performed by means of the thermometer molecule p-methoxybenzylpyridinium chloride [23]. This compound exhibits a simple fragmentation pattern with the p-methoxybenzyl cation being the only intense fragment ion. The peak area ratio of the fragment ion and the sum of intact precursor- and fragment ion in combination with Rice-RamspergerKassel-Marcus calculation of the rate coefficients for the unimolecular decomposition allow for an estimation of the internal ion energy [23]. Almost all detected effective temperatures were in the reliable range from about 800 to $1500 \mathrm{~K}$. All measurements for determination of the effective plume temperature were repeated six times, each consisting of 40 shots $\times 30$ subspectra. For every measurement a new sample spot was used. The areas of matrix ions with identical $\mathrm{m} / \mathrm{z}$ values to the thermometer parent ion $\mathrm{M}^{+}$or its fragment ion $\mathrm{F}^{+}$were typically below $10 \%$ of the corresponding peak areas of $\mathrm{M}^{+}$or $\mathrm{F}^{+}$at a laser fluence optimized for highest analyte $\mathrm{S} / \mathrm{N}$ values. Nevertheless, they were taken into account by subtracting the matrix areas from the peak areas of $\mathrm{M}^{+}$and $\mathrm{F}^{+}$by measuring pure DD or sublimed matrix spots using identical device parameters. The lower and upper limits of the effective temperature determinations were defined from $\mathrm{M}^{+}$or $\mathrm{F}^{+}$intensities close to the detection limit and maximum tolerable matrix ion intensities.

\section{Results and Discussion}

\section{Crystal Size}

The particle size and morphology of DD and sublimed preparations of the cinnamic acid derivatives $\mathrm{CHCA}$, 4-HO-3-Me-CCA, 4-HO-3-MeO-CCA, 4-Me-CCA, and SA (Figure 1) were determined by SEM [Figure 2 and SI Figure 6 (large)]. In the case of sublimed preparations, SEM micrographs were recorded after the surfaces have been partially redissolved with $0.5 \mu \mathrm{L} \mathrm{30 \%} \mathrm{ACN/0.01 \%}$ TFA and recrystallized. The remaining solid matrix acts most probably as a seed layer during recrystallization, therefore no change in crystal morphology was detected in comparison to micrographs recorded before, also using lower or higher solvent amounts or ACN concentrations up to $50 \%$ (SI Figure 7) [13, 14]. Sub-

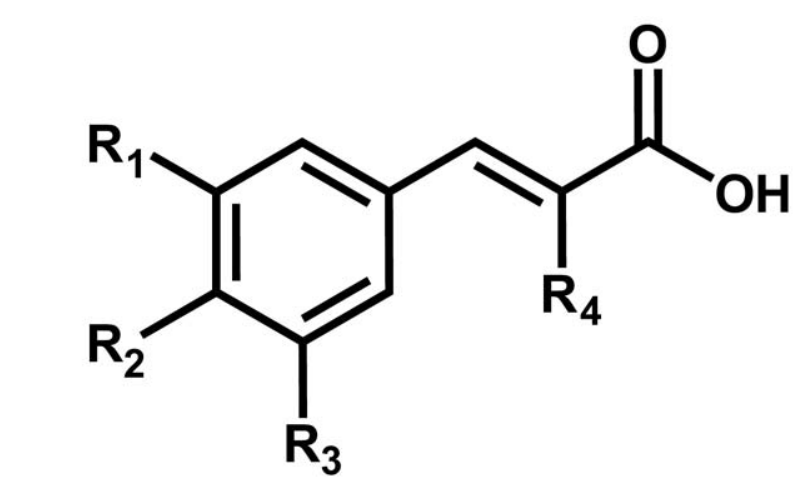

\begin{tabular}{|c|c|c|c|c|}
\hline matrix & $\mathbf{R}_{\mathbf{1}}$ & $\mathbf{R}_{\mathbf{2}}$ & $\mathbf{R}_{\mathbf{3}}$ & $\mathbf{R}_{\mathbf{4}}$ \\
\hline $\mathrm{CHCA}$ & $\mathrm{H}$ & $\mathrm{OH}$ & $\mathrm{H}$ & $\mathrm{CN}$ \\
\hline $\mathrm{Me}-\mathrm{CCA}$ & $\mathrm{H}$ & $\mathrm{CH}_{3}$ & $\mathrm{H}$ & $\mathrm{CN}$ \\
\hline 4-HO-3-Me-CCA & $\mathrm{CH}_{3}$ & $\mathrm{OH}$ & $\mathrm{H}$ & $\mathrm{CN}$ \\
\hline 4-HO-3-MeO-CCA & $\mathrm{OCH}_{3}$ & $\mathrm{OH}$ & $\mathrm{H}$ & $\mathrm{CN}$ \\
\hline $\mathrm{SA}$ & $\mathrm{OCH}_{3}$ & $\mathrm{OH}$ & $\mathrm{OCH}_{3}$ & $\mathrm{H}$ \\
\hline
\end{tabular}

Figure 1. Chemical structures of the used cinnamic acid derivatives.

limed CHCA forms compact crystals with typical dimensions of about $100 \mathrm{~nm}$ and a narrow size distribution (Figure 2a, right). CHCA crystals prepared by DD preparation exhibit dimensions of some micrometers and are therefore more than one order of magnitude larger (Figure 2a, left). SA and 4-Me-CCA also exhibit significantly decreased crystal sizes in the nanometer range when sublimed compared with micrometer sized crystals for DD preparations (Figure 2b, c). By contrast, crystal structures of 4-HO-3-Me-CCA and 4-HO-3$\mathrm{MeO}-\mathrm{CCA}$ consist of sub-micrometer dimensions for both sublimed and DD spots (Figure 2d and e small). All tested compounds form macroscopic structures for DD preparations (Figure 2 left) whereas a homogeneous distribution can be observed after sublimation (Figure 2 right).

\section{Effective Temperature}

Heat conduction simulations upon laser irradiation in [24] have demonstrated that the surface temperature of small-size matrix crystals is significantly higher compared with larger crystals. This effect was attributed to the hindrance of heat dissipation across phase interfaces, which is more pronounced in small crystals thus leading to higher temperatures [24]. The model is qualitatively in accordance with the microscopic observation that small crystals evaporate at lower laser fluences than larger crystals in the order of micrometer, the latter exhibiting layer-by-layer evaporation [24]. In addition, the temperature-size dependence of the model in [24] reveals a steep temperature dependence on the crystal size for sub-micron particles, whereas a very 


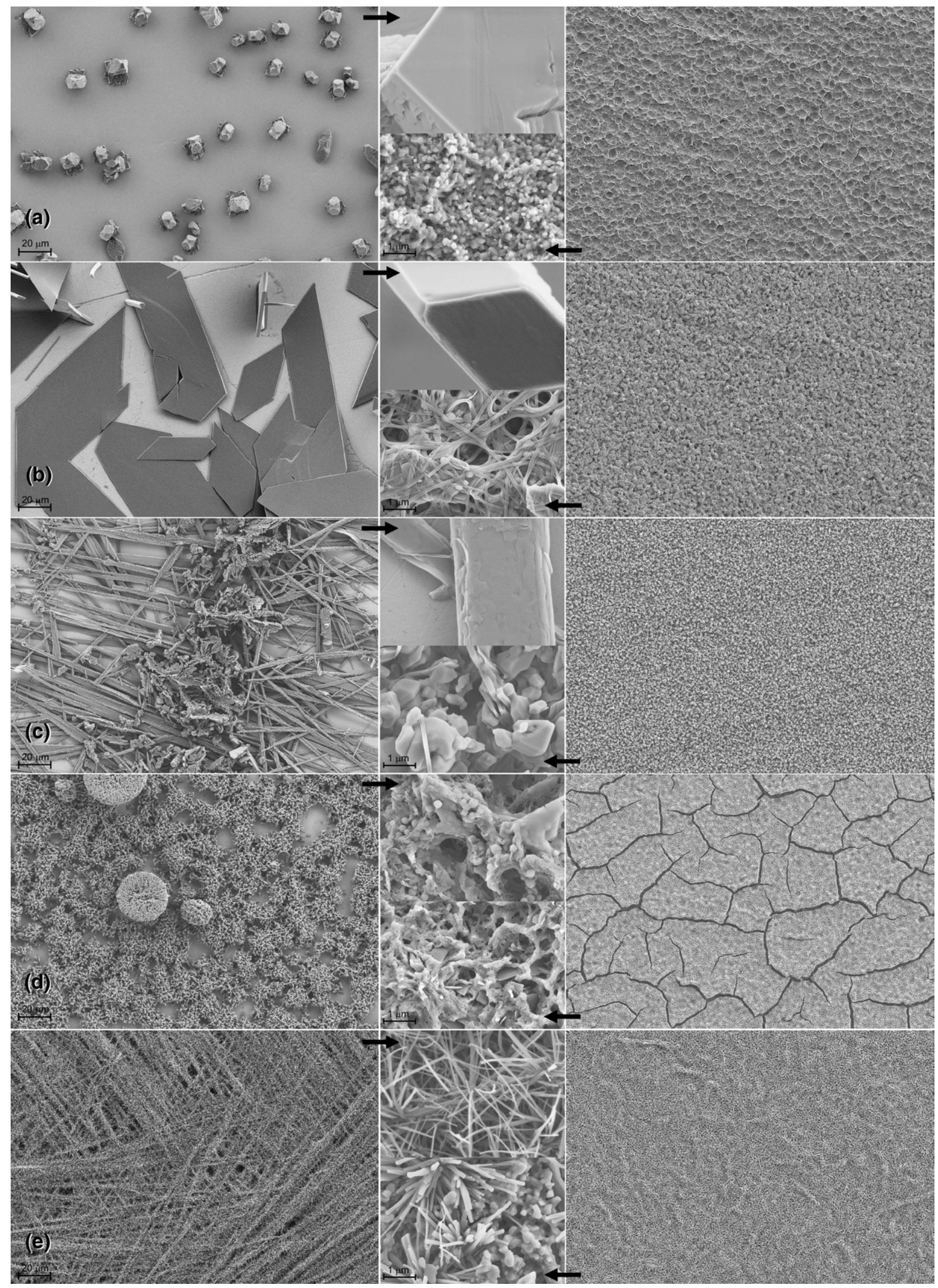

Figure 2. Matrix crystals resulting from standard DD preparations on stainless steel plate (left) and from sublimed preparations on hydrophobically coated aluminum oxide surfaces (right) shown in $500 \times$ (large pictures) and 20,000 $\times$ magnifications (small pictures). From upper to lower: CHCA (a), $\mathrm{SA}(\mathbf{b}), \mathrm{Me}-\mathrm{CCA}(\mathbf{c}), 4-\mathrm{HO}-3-\mathrm{Me}-\mathrm{CCA}(\mathbf{d})$, and 4-HO-3-MeO-CCA (e). 
shallow temperature dependence is obtained for particle dimensions larger than a few microns. We therefore speculate that crystal structures of CHCA, SA, and 4-Me-CCA prepared by sublimation are small enough to obstruct heat conduction. As a consequence, transient superheating with much higher temperatures compared with their larger sized DD counterparts are expected. Measurements of the effective temperatures of these compounds indeed reveal about $100 \mathrm{~K}$ higher temperatures for sublimed CHCA and 4-Me-CCA at pulse energies for highest analyte $\mathrm{S} / \mathrm{N}$ ratios (Figure 3). In the case of sublimed SA and low fluences, the effective temperature is more than $200 \mathrm{~K}$ higher than for DD preparations, whereas an unexpected and not explainable temperature drop with a minimum at about $10.5 \mu \mathrm{J} /$ pulse was detected at higher laser fluences (Figure 3). 4-HO-3-Me-CCA and 4-HO-3-MeO-CCA exhibit crystal structures of about the same size for DD and sublimed preparations (Figure $2 \mathrm{~d}$, e). Therefore, the same effective temperature for both preparation methods is expected, which is observed in the experiment (Figure 3) and supports the qualitative model of a crystal size-dependent plume temperature.

\section{MS Intensities}

For the comparison of the analyte ion signal intensities between sublimed and DD preparations, a standard peptide mix and laser fluence range to obtain maximum analyte signal intensity for each matrix were applied. Figure 4 shows peptide signals within a $\mathrm{m} / \mathrm{z}$ range from 1000 to 2000 Da obtained from 500 amol of a BSA in-solution digest using CHCA as matrix. The spectra show that the sublimed preparation yields clearly more analytes and higher analyte ion intensities compared with DD preparations. In addition, the spectral noise obtained with sublimed spots was somewhat lower compared with DD preparations. As an example, the baselines account for about $15 \%$ (sublimed) and $16 \%$ (DD) at $1000 \mathrm{Da}$, for about 3.5\% (sublimed) and 7\% (DD) at $1500 \mathrm{Da}$, and for about 2\% (sublimed) and $3 \%$ (DD) at $2000 \mathrm{Da}$ of the strongest analyte peaks (Figure 4). After acquiring sum spectra consisting of four replicate experiments for each preparation method and applying stringent selection criteria, which were internal calibration and restriction to consistently observed signals within a mass tolerance of $20 \mathrm{ppm}$, only 11 tryptic peptides were detectable for the DD preparation as opposed to 30 peptides for the sublimed spots (without consideration of cystines or peptides generated by combined tryptic and chymotryptic activity), for details see SI Table 2. The sequence coverage is thus raised to $46 \%$ compared with $17 \%$ for the DD preparation. Absolute ion intensities as well as peak areas and especially $\mathrm{S} / \mathrm{N}$ ratios of the tryptic peptides which were detectable with both preparation techniques were also increased by averaged factors of 4.6 (intensity), 6.5 (area), and 19.9 (S/N) when sublimed spots were used.
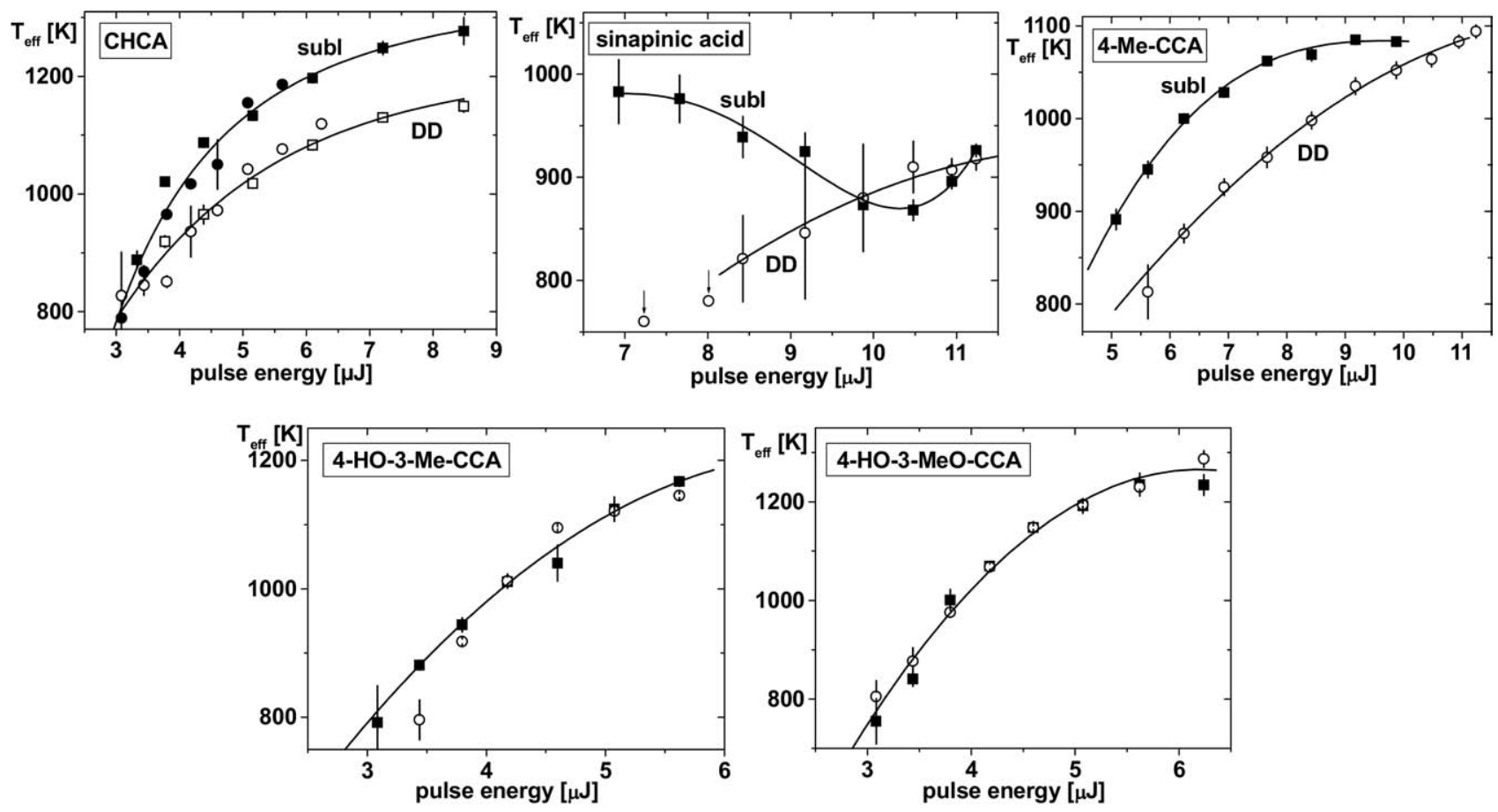

Figure 3. Effective temperatures of p-methoxybenzylpyridinium chloride in the matrix compounds CHCA, SA, 4-Me-CCA, 4-HO-3-Me-CCA, and 4-HO-3-MeO-CCA for DD (open) and sublimed preparations (filled). The amount of thermometer molecule was $0.5 \mu \mathrm{L}$ of a $2 \mathrm{pmol} / \mu \mathrm{L}$ solution in all cases except for CHCA for which an additional amount of $4 \mathrm{pmol} / \mu \mathrm{L}$ was used (open squares for DD, and filled circles for sublimed spots, respectively). 


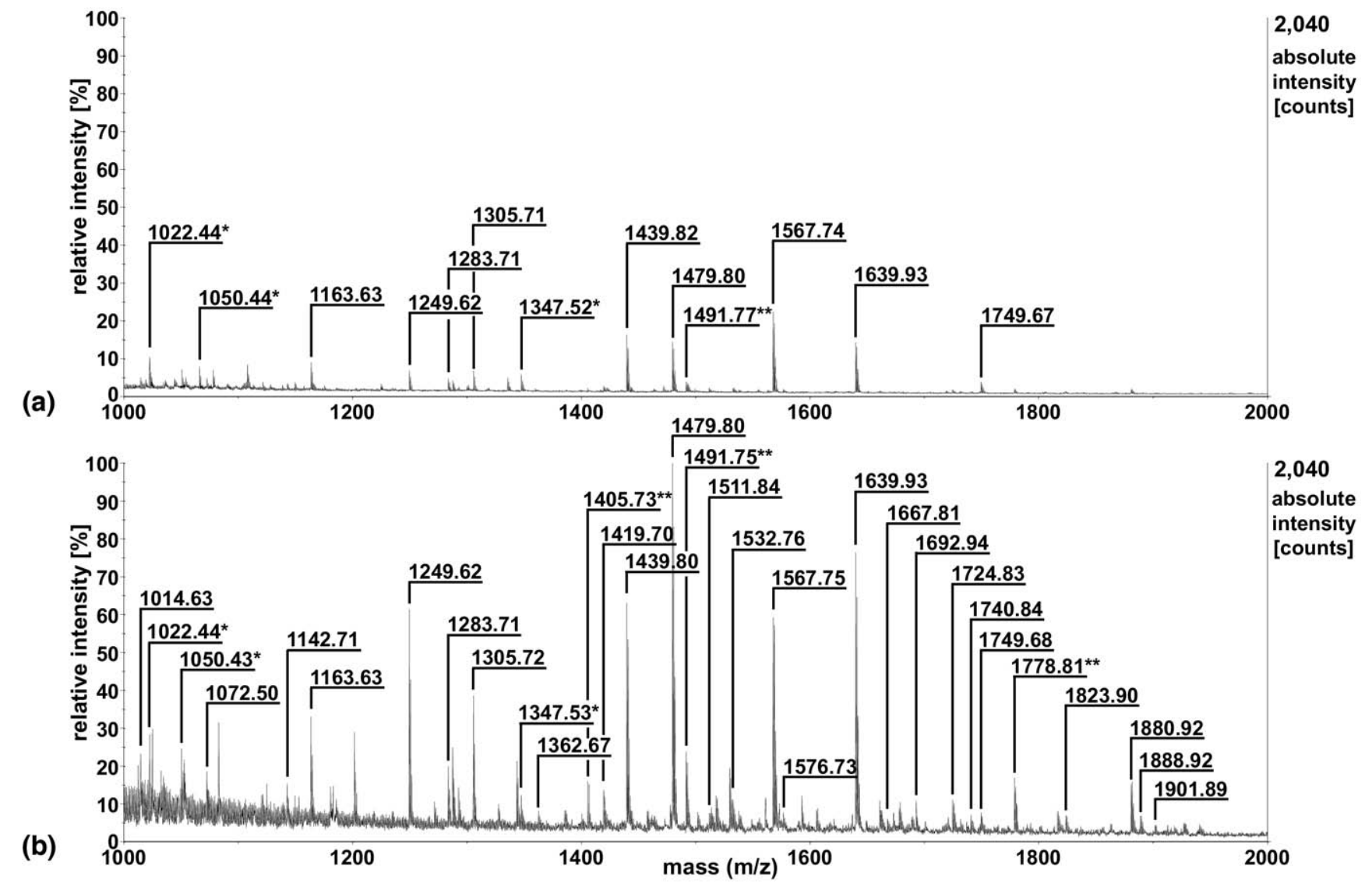

Figure 4. MS sum spectra ranging from 1000 to 2000 Da for a tryptic in-solution digest of BSA using CHCA as DD (a) or sublimed (b) preparation. Mass numbers of assigned BSA peptides are given only for consistently observed signals within four spectra with 1200 shots each. The total sample load referring to BSA was 500 amol. Signals with one asterisk refer to cystine peptides and with two asterisks to peptides with combined tryptic and chymotryptic activity.

The increase in analyte sensitivity of sublimed matrix compounds with higher effective temperatures than their DD counterparts is further shown for the used matrices and des-arg ${ }^{1}$-bradykinin as analyte in Figure 5. Identical acquisition parameters were used both for sublimed and DD preparations. When CHCA is used as matrix we noticed that the signal peak areas do not get saturated upon increasing laser fluence but peak at a certain laser fluence, e.g., between 5.5 and $6 \mu \mathrm{J} /$ pulse for sublimed spots (Figure 5, CHCA). As a likely reason, we suggest an increased analyte fragmentation upon very high pulse energies (Figure 3, CHCA), which probably overcompensates a further increase in analyte intensity. The effective pulse energy at the optimum laser fluence for sublimed CHCA preparations was detected at about 5.5-6 $\mathrm{\mu J} /$ pulse (Figure 5, CHCA), which correlates with an effective temperature of about 1150-1200 K (Figure 3, CHCA). This effective temperature was reached at about $8-9 \mu \mathrm{J} /$ pulse for the corresponding DD preparation (Figure 3, CHCA). At this laser fluence the DD preparation also exhibited a maximum in analyte ion area, which, however, was less distinct (Figure 5, CHCA). As a practical consequence, the optimum laser fluence has to be chosen carefully to achieve maximum sensitivity. Sublimed matrices that do not yield higher effective temperatures compared with their DD counterparts also did not exhibit higher analyte ion intensities over the entire fluence range, as shown for 4-HO-3-Me-CCA and 4-HO-3-MeO-CCA in Figure 5. However, for 4-HO-3-MeO-CCA, a difference of almost one order of magnitude between the two preparation methods was found for higher laser energies (Figure 5). We therefore assume that higher effective temperatures obtained at smaller crystal structures are the primary but not exclusive cause for the detected higher analyte signal intensities because of a more efficient volatilization.

The strong enhancement in MS performance is further depicted for CHCA and different peptides in SI Figure 8. An increase in analyte ion area of about one order of magnitude was detected for all analytes, independent of their mass. Again, the analyte ion signal areas peaked at a certain laser fluence for sublimed preparations. In addition, a higher shot-to-shot reproducibility of analyte signals generated by sublimed spots could be detected, which resulted in much smaller standard deviations. 

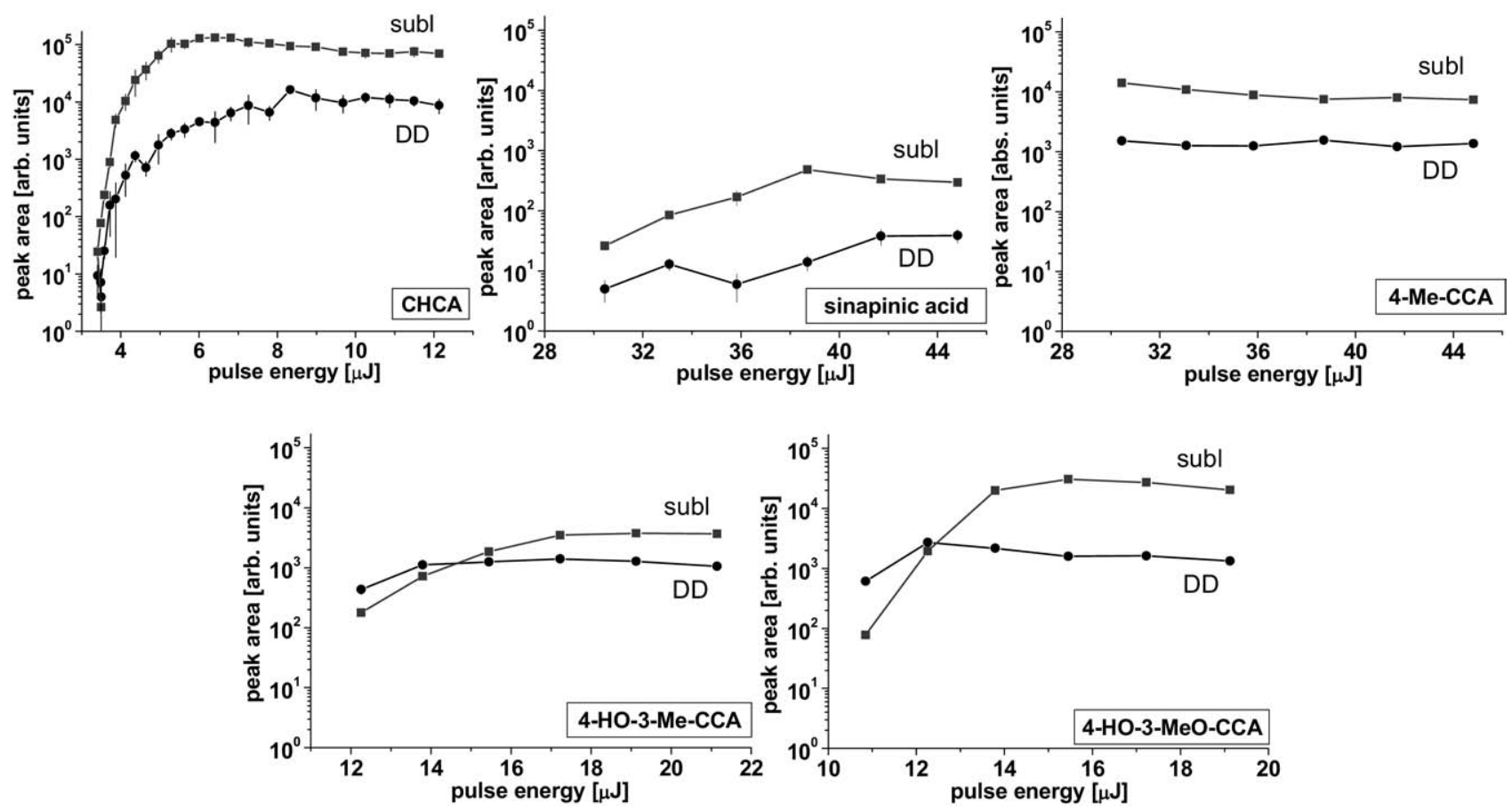

Figure 5. Detected peak areas for $62.5 \mathrm{fmol}$ of des-arg ${ }^{1}$-bradykinin presented on a logarithmic scale with identical scaling for sublimed preparations (squares) and DD preparations (circles).

\section{Initial Ion Velocity}

Studies of the initial ion velocity in MALDI have been presented in [25] as a valuable technique to differentiate between different MALDI desorption processes. Here we apply this technique to further clarify possible differences between the ion formation processes of sublimed and DD preparations. Theoretical investigations predict two desorption mechanisms that occur in MALDI: thermal vaporization of single molecules up to small clusters consisting of up to four molecules and phase explosions with ablation of large clusters at higher laser fluences [26-28]. It was shown that the process of thermal vaporization of small molecules gets favored by increase of the effective temperature, which is accompanied by lower initial ion velocities [22]. Therefore, the question rose whether higher matrix crystal temperatures caused from smaller crystals accompanied by higher effective analyte temperatures in sublimed matrices cause more efficient thermal vaporization by heating and vaporization of the matrix in a larger volume or increased phase explosions by stronger ablation processes. The mean initial ion velocities of protonated bovine insulin were measured using the $\mathrm{DE}$ method [29] from the different matrix compounds, each prepared as DD and sublimed spots. The DE method only allows for determination of the average axial velocity of ions without separation of parameters as width and overall shape of the velocity distribution [29, 30]. The detected velocities measured for the DD preparations were found to be in good agreement with the values known from the literature [29, 30]. For all sublimed compounds a strong increase in the initial ion velocity of about factor 2 was measured, e.g., $522 \pm 4$ $\mathrm{m} / \mathrm{s}$ for CHCA compared with $250 \pm 3 \mathrm{~m} / \mathrm{s}$ for the correlating DD preparation (Table 1). The detected higher initial ion velocities suggest that no increased thermal evaporation of single molecules but increased phase explosion processes with ablation of larger clusters occur, resulting in enhanced intensities also for large, not thermally evaporable analytes. This is in agreement with the enhanced detection of large mass analytes compared with identical DD approaches (SI Table 2). As a practical consequence of significantly different initial ion velocities, the initial velocity parameter in the instrumental settings should be adapted to achieve the highest possible MS/MS mass accuracy.

Table 1. Initial velocities of protonated bovine insulin compared between DD and vacuum sublimed preparations for the used cinnamic acid derivatives. Sublimed samples exhibit a strong increase in initial ion velocity compared to the correlating DD preparations

\begin{tabular}{|c|c|c|c|c|c|c|c|c|c|c|}
\hline Matrix & & & & & 4-M & $-\mathrm{CCA}$ & $4-\mathrm{HO}-3$ & Me-CCA & 4-HO-3-I & eO-CCA \\
\hline Preparation & & & & Initi & velocity & (insulin + & -1) $)^{+}[\mathrm{m} / \mathrm{s}]$ & & & \\
\hline DD sublimed & $250 \pm 3$ & $522 \pm 4$ & $359 \pm 8$ & $475 \pm 7$ & $187 \pm 9$ & $370 \pm 19$ & $239 \pm 34$ & $461 \pm 11$ & $278 \pm 16$ & $416 \pm 7$ \\
\hline
\end{tabular}


Higher initial ion velocities are found to correlate with lower internal energies and hence with lower fragmentation efficiencies, probably due to cooling processes upon expansion of the matrix plume [25, 26, 30]. In comparison to DD preparations, MS/MS fragments of tryptic BSA peptides spotted on sublimed matrices with reduced crystal sizes indeed exhibited less increased intensities than the analytes detected in MS measurements (data not shown). Nevertheless, complete evaporation of microcrystals as well as analyte accumulation in the uppermost matrix layers due to the used surface preparations (discussed in more detail below) can explain, why slightly higher MS/MS-based peptide scores from the sublimed matrix preparations can be detected in spite of stronger cooling effects [17].

The reason for the detected higher initial ion velocity values is not yet fully understood. Glückmann and Karas have shown that inhomogeneities in crystal morphology within one sample usually correlate with differences in initial ion velocities, which are too large to be attributed to different ion starting positions [30]. Furthermore, the addition of co-matrices to sample preparations has impact on the initial ion velocity most likely due to altered sample homogeneities [30]. Therefore, it can be assumed that the uniform matrix deposition of all sublimed matrices, which can be seen by means of the homogeneous macroscopic crystal morphologies (Figure 2), might cause this effect.

\section{Analyte Incorporation Depth}

The higher effective temperatures of vacuum sublimed matrix preparations with significantly smaller crystal sizes compared with DD preparations probably cause complete volatilization of the irradiated crystals, which seems to be mainly responsible for the increase in analyte ion intensity. Apart from this, a further advantage of sublimed matrix preparations is a lateral analyte accumulation caused by the hydrophobic surface which focuses the droplet on a small spot size. Additionally, the surface preparation causes a vertical analyte concentration with analyte incorporation only in the uppermost matrix crystal layers. Both effects yield high analyte-to-matrix ratios and could be responsible for the detected increase in analyte ion intensity at higher laser fluences for sublimed 4-HO-3-Me-CCA and 4-HO3-MeO-CCA compared with their DD counterparts (Figure 5), since these matrix compounds did not exhibit reduced crystal sizes upon sublimation (Figure $2 \mathrm{~d}$, and e small). The vertical analyte distribution in the matrix crystals was further investigated by analyzing the average laser penetration depth of sublimed CHCA samples, which was determined to $6.4 \pm 1.5 \mathrm{~nm}$ per shot using a wavelength of $355 \mathrm{~nm}$ and fluences optimized for highest $\mathrm{S} / \mathrm{N}$ ratios (see SI Figure 3 and Experimental Section) as well as the following analyte depth profile: The surfaces of sublimed and DD spots, using $\mathrm{CHCA}$ and standard peptides as matrix and analytes, respectively, were ablated layer-by-layer by means of only a few laser shots per irradiation cycle. For the purpose of generating identical spot size diameters and identical overall matrix-to-analyte ratios for DD prepared control experiments, DD spots were prepared by completely dissolving fresh and unused sublimed matrix spots with $0.5 \mu \mathrm{L} \mathrm{70 \%} \mathrm{ACN,} \mathrm{see} \mathrm{SI} \mathrm{Figure} \mathrm{9.} \mathrm{Two}$ approaches were accomplished: first, "drilling" at fixed spot positions with sequences of 20 shots each and second, irradiation of the complete spot surface with sequences of $25 \times 20$ shots with subsequent random changes after each 20 shot sub-spectrum. The first approach allows for a statement about the analyte incorporation depth at a single location with the disadvantage of spatial inhomogeneities in analyte incorporation, such as the known "hot spot" phenomenon. The second approach delivers an average value of the analyte incorporation by homogeneous layer-by-layer ablation of the complete spot surface; therefore fluctuations due to "hot spots" are eliminated. Disadvantages of this approach are a possibly non-uniform irradiation of the spot surface delivering analyte ions from different matrix depths and possible sample redepositions in regions around a laser shot, which will lead to false results. Both approaches were repeated six times each and revealed nearly identical results, see Figure 6 (local "drilling") and SI Figure 10 (homogeneous ablation). After each penetration cycle the $\mathrm{S} / \mathrm{N}$ values of the analytes, which were incorporated in the respective ablated layer, were determined and referred to the correlating analyte $\mathrm{S} / \mathrm{N}$ values obtained from the ablation of the first layer. Within the homogeneous ablation approach, more than one penetration cycle is necessary for complete ablation of one matrix layer, since only roughly $3 \%$ of the spot surface is irradiated per shot (spot diameter: $600 \mu \mathrm{m}$, laser spot diameter: $\sim 100 \mu \mathrm{m}$ ). Therefore, more penetration cycles are necessary for reaching the same depth as for the "drilling" approach, which explains the higher number of laser penetration cycles; compare Figure 6 and SI Figure 10. This stronger ablation per shot for the "drilling" approach might also explain the missing analyte $\mathrm{S} / \mathrm{N}$ maxima of the sublimed samples. Surface prepared sublimed matrix spots exhibited a much stronger analyte accumulation in the outer crystal layers compared with DD preparations, since it is not possible to incorporate analytes into the already existing deeper crystal regions. This general trend applies for all tested analytes, independently of their particular $m / z$ ratio. The near surface accumulation of the analyte causes higher analyte-to-matrix ratios of the ablated analyte-containing matrix layers and therefore enables for the detection of stronger analyte ion signal intensities. For the "drilling" approach with stronger ablation at fixed locations matrix signals were still intense after 21 irradiation cycles, and no complete ablation could be detected using optical microscopy. This further indicates analyte incorporation into the uppermost matrix layers of sublimed samples.

Furthermore, a correlation between the analyte mass and the depth of the strongest analyte incorporation 


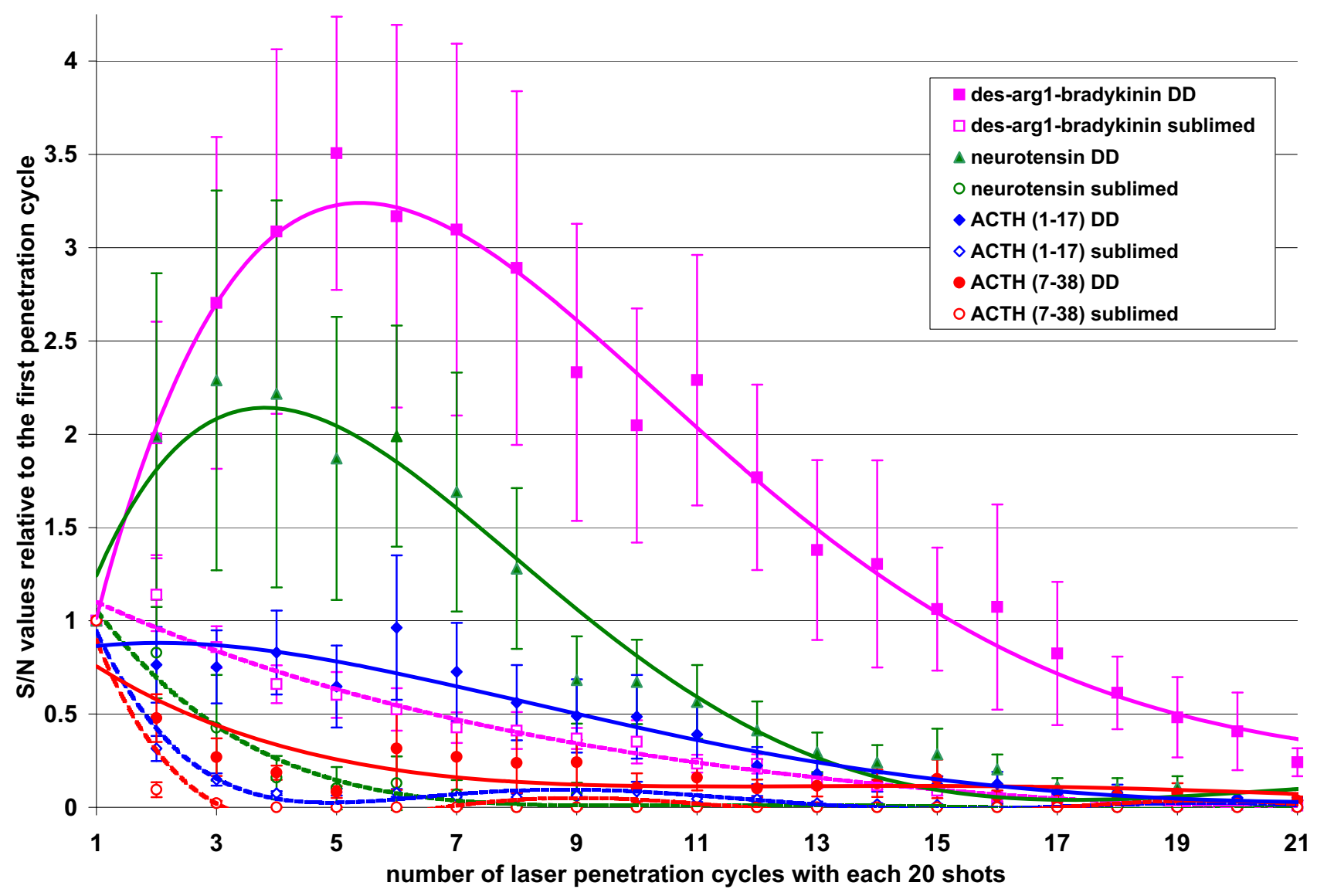

Figure 6. S/N ratios of peptides of various masses in dependency on the number of laser penetration cycles with laser irradiation at fixed spot positions. The analyte $\mathrm{S} / \mathrm{N}$ ratios are normalized by the value of the first layer.

could be detected for both preparations and both experimental approaches: Low mass analytes, such as des-arg'-bradykinin (903 Da), angiotensin I (1296 Da), or neurotensin (1673) exhibited their highest S/N values and therefore their strongest incorporation in deeper crystal regions, while higher mass analytes, such as ACTH (7-38) (3657 Da), are nearly quantitatively concentrated in the uppermost matrix layers (Figure 6, SI Figure 10). Solubility effects by which hydrophilic analytes remain longer dissolved than hydrophobic ones resulting in a solubility dependent incorporation sequence might explain this observation. However, no correlation was found between the GRAVY score as an indicator for the analyte hydrophobicity/hydrophilicity and the incorporation of the analytes, also taking into account that at acidic $\mathrm{pH}$ values acidic functionalities are protonated and therefore less hydrophilic. Another explanation concerns a lower heat of adsorption for the incorporation of high mass analytes, as the resulting crystal defects will be more unfavorable than in the case of smaller analytes.

An increase of the ACN percentage or the total volume of the analyte solution decreased the analyte peak areas per ablated matrix layer of sublimed preparations, which can be attributed to a higher amount of dissolving matrix that enables analyte incorporation also in deeper regions of the crystal [13].

\section{Conclusions}

Sublimed matrix spots enable the handling of extremely low and precisely positioned matrix amounts. The use of hydrophobic coatings surrounding the matrix spots enables to confine the analyte solution on a small matrix spot surface $\left(<0.3 \mathrm{~mm}^{2}\right)$. In combination with surface preparations high analyte-to-matrix ratios in the outer crystal layers are achievable. Therefore, higher analyte amounts can be ablated per laser shot with the result of raised analyte sensitivity.

Some of the tested compounds, such as sublimed CHCA, show reduction of the matrix particle size to dimensions probably small enough to obstruct heat conduction, which is supposed to be the reason for the raising temperature in the crystals upon laser irradiation. In these cases, strongly enhanced analyte intensities can be achieved, probably caused by more effective ablation-assisted volatilization. The detected higher initial velocities of analytes in sublimed preparations probably lower the internal ion energy with the beneficial effect of higher stability of the desorbed analytes 
compared with standard DD preparations, which can be an additional reason for the detected increase in analyte intensity. Increased analyte ion yields from very thin electrosprayed matrix samples have been reported [31]. According to a model from Knochenmuss et al., this was explained by an interaction between matrix molecule and metal substrate electronic states facilitating a two-photon ionization process instead of three photons as needed for the bulk matrix [32]. Similar processes for the sublimed matrix layers as explanation for the detected increase in analyte ion intensities can be excluded, since no direct contact between matrix and metal is possible due to the hydrophobic coating of the substrate surface before matrix sublimation. The analyte signal intensity exhibits a maximum with increasing laser fluence when CHCA is used as matrix, which probably can be due to contrary effects of increasing analyte ablation and ionization on the one hand and increasing fragmentation efficiency on the other.

\section{Acknowledgments}

The authors are grateful for the excellent technical assistance of A. Mayr and C. Macioschek (Qiagen N.V.).

The invention of 4-Me-CCA as MALDI matrix was filed as a German patent.

T. W. Jaskolla, M. Karas, and K. Reihs declare that they have no competing financial interests.

\section{Appendix A Supplementary Material}

Supplementary material associated with this article may be found in the online version at doi:10.1016/ j.jasms.2009.02.010.

\section{References}

1. Karas, M.; Bachmann, D.; Hillenkamp, F. The Influence of the Wavelength in High Irradiance Ultraviolet Laser Desorption Mass Spectrometry of Organic Molecules. Anal. Chem. 1985, 57, 2935-2939.

2. Karas, M.; Bachmann, D.; Bahr, U.; Hillenkamp, F. Matrix-assisted Ultraviolet Laser Desorption of Nonvolatile Compounds. Int. J. Mass Spectrom. Ion Processes 1987, 78, 53-56.

3. Karas, M.; Hillenkamp, F. Laser Desorption Ionization of Proteins with Molecular Masses Exceeding 10,000 Dalton. Anal. Chem. 1988, 60, 2299-2301.

4. Miliotis, T.; Kjellström, S.; Nilsson, J.; Laurell, T.; Edholm, L.-E.; MarkoVarga, G. Ready-Made Matrix-Assisted Laser Desorption/Ionization Target Plates Coated with Thin Matrix Layer for Automated Sample Deposition in High-Density Array Format. Rapid Commun. Mass Spectrom. 2002, 16, 117-126.

5. Haddleton, D. M.; Waterson, C.; Derrick, P. J. Comment: A Simple, Low-Cost, Air-Spray Method for Improved Sample Preparation for Matrix-Assisted Laser Desorption/Ionization Mass Spectrometry of Derivatized Poly(Ethylene Glycol). Eur. Mass Spectrom. 1998, 4, 203-207.

6. Axelsson, J.; Hoberg, A.-M.; Waterson, C.; Myatt, P.; Shield, G. L.; Varney, J.; Haddleton, D. M.; Derrick, P. J. Improved Reproducibility and Increased Signal Intensity in Matrix-Assisted Laser Desorption/ Ionization as a Result of Electrospray Sample Preparation. Rapid Commun. Mass Spectrom. 1997, 11, 209-213.

7. Preisler, J.; Foret, F.; Karger, B. L. On-Line MALDI-TOF MS Using a Continuous Vacuum Deposition Interface. Anal. Chem. 1998, 70, 52785287.

8. Preisler, J.; Hu, P.; Rejtar, T.; Karger, B. L. Capillary ElectrophoresisMatrix-Assisted Laser Desorption/Ionization Time-of-Flight Mass Spectrometry Using a Vacuum Deposition Interface. Anal. Chem. 2000, 72, 4785-4795.
9. Preisler, J.; Hu, P.; Rejtar, T.; Moskovets, E.; Karger, B. L. Capillary Array Electrophoresis-MALDI Mass Spectrometry Using a Vacuum Deposition Interface. Anal. Chem. 2002, 74, 17-25.

10. Hankin, J. A.; Barkley, R. M.; Murphy, R. C. Sublimation as a Method of Matrix Application for Mass Spectrometric Imaging. J. Am. Soc. Mass Spectrom. 2007, 18, 1646-1652.

11. Reihs, K.; Engelking, J.; Harder, P.; Nordhoff, E.; Röhl, H.; Rudakowski, S.; Vorberg, K.; Wesener, J. Ready-Made MALDI Target Plates Containing High-Density Arrays of Vacuum-Deposited Matrix Spots on Ultraphobic Surfaces. Proceedings of the 51st ASMS Conference on Mass Spectrometry and Allied Topics; Montreal, Canada, June, 2003.

12. Muscate-Magnussen, A.; Zhu, X.; Wesener, J.; Röhl, H.; Harder, P. Reihs, K. A New Approach of Liquid Sample Handling on MALDI Plates Enabling Advanced High Throughput Proteomics Applications. Proceedings of the 52nd ASMS Conference on Mass Spectrometry and Allied Topics; Nashville, TN, May, 2004.

13. Röhl, H.; Goethel, S.; Pickartz, J.; Wesener, J.; Reihs, K. Depth Profiling of CHCA Matrix Spots for the Investigation of Sensitivity and Dynamic Range of Peptide Analysis by MALDI-TOF MS. Proceedings of the 53rd ASMS Conference on Mass Spectrometry and Allied Topics; San Antonio, TX, June, 2005.

14. Röhl, H.; Goethel, S.; Reihs, K. MPep MALDI Chips for High-Sensitivity and High-Throughput Peptide Analysis by MALDI-TOF MS. Nature Methods 2005, 2, 6 (Application Note).

15. Jaskolla, T.; Roth, U.; Röhl, H.; Glückmann, M.; Karas, M.; Steinert, K.; Reihs, K. Sensitive and Reproducible Detection of Peptides by MALDITOF MS Using Self-Assembled Monolayer (SAM)-Based ChipsSpecifications and Applications. Proceedings of the 54th ASMS Conference on Mass Spectrometry and Allied Topics; Seattle, WA, May, 2006.

16. Jaskolla, T.; Karas, M.; Steinert, K.; Roth, U.; Menzel, C.; Reihs, K. Effects of Matrix Crystal Size on Ion Formation in Matrix-Assisted Laser Desorption/Ionization. Proceedings of the 55th ASMS Conference on Mass Spectrometry and Allied Topics; Indianapolis, IN, June, 2007.

17. Poetsch, A.; Schlüsener, D.; Florizone, C.; Eltis, L.; Menzel, C.; Rögner, M.; Steinert, K.; Roth, U. Improved Identification of Membrane Proteins by MALDI-TOF MS/MS Using Vacuum Sublimated Matrix Spots on an Ultraphobic Chip Surface. J. Biomol. Tech. 2008, 19, 129-138.

18. Önnerfjord, P.; Ekström, S.; Bergquist, J.; Nilsson, J.; Laurell, T.; MarkoVarga, G. Homogeneous Sample Preparation for Automated High Throughput Analysis with Matrix-assisted Laser Desorption/Ionization Time-of-Flight Mass Spectrometry. Rapid Commun. Mass Spectrom. 1999, 13, 315-322.

19. Gabelica, V.; Schulz, E.; Karas, M. Internal Energy Build-Up in MatrixAssisted Laser Desorption/Ionization. J. Mass Spectrom. 2004, 39, 579 593.

20. Jaskolla, T. W.; Lehmann, W. D.; Karas, M. 4-Chloro- $\alpha$-Cyanocinnamic Acid Is an Advanced, Rationally Designed MALDI Matrix. Proc. Natl. Acad. Sci. U.S.A. 2008, 105, 12200-12205.

21. Owen, S. J.; Meier, F. S.; Brombacher, S.; Volmer, D. Increasing Sensitivity and Decreasing Spot Size Using an Inexpensive, Removable Hydrophobic Coating for Matrix-Assisted Laser Desorption/Ionization Plates. Rapid Commun. Mass Spectrom. 2003, 17, 2439-2449.

22. Jaskolla, T. W.; Karas, M. Using Fluorescence Dyes as a Tool for Analyzing the MALDI Process. J. Am. Soc. Mass Spectrom. 2008, 19, 1054-1061.

23. Luo G.; Marginean I.; Vertes, A. Internal Energy of Ions Generated by Matrix-Assisted Laser Desorption/Ionization. Anal. Chem. 2002, 74, 6185-6190

24. Sadeghi M.; Vertes A. Crystallite Size Dependence of Volatilization in Matrix-Assisted Laser Desorption Ionization. Appl. Surf. Sci. 1998, 127/129, 226-234.

25. Karas, M.; Bahr, U.; Fournier, I.; Glückmann, M.; Pfenniger, A. The Initial-Ion Velocity as a Marker for Different Desorption-Ionization Mechanisms in MALDI. Int. J. Mass Spectrom. 2003, 226, 239-248.

26. Zhigilei, L. V.; Kodali, P. B. S.; Garrison, B. J. Molecular Dynamics Model for Laser Ablation and Desorption of Organic Solids. J. Phys. Chem. B 1997, 101, 2028-2037.

27. Zhigilei, L. V.; Kodali, P. B. S.; Garrison, B. J. A Microscopic View of Laser Ablation. J. Phys. Chem. B 1998, 102, 2845-2853.

28. Zhigilei, L. V.; Garrison, B. J. Microscopic Mechanisms of Laser Ablation of Organic Solids in the Thermal and Stress Confinement Irradiation Regimes. J. Appl. Phys. 2000, 88, 1281-1298.

29. Juhasz, P.; Vestal, M. L.; Martin, S. A. On the Initial Velocity of Ions Generated by Matrix-Assisted Laser Desorption Ionization and its Effect on the Calibration of Delayed Extraction Time-of-Flight Mass Spectra. J. Am. Soc. Mass Spectrom. 1997, 8, 209-217.

30. Glückmann, M.; Karas, M. The Initial Ion Velocity and its Dependence on Matrix, Analyte, and Preparation Method in Ultraviolet MatrixAssisted Laser Desorption/Ionization. J. Mass Spectrom. 1999, 34, 467477.

31. McCombie, G.; Knochenmuss, R. Enhanced MALDI Ionization Efficiency at the Metal-Matrix Interface: Practical and Mechanistic Consequences of Sample Thickness and Preparation Method. J. Am. Soc. Mass Spectrom. 2006, 17, 737-745.

32. Knochenmuss, R.; McCombie, G.; Faderl, M. Ion Yields of Thin MALDI Samples: Dependence on Matrix and Metal Substrate and Implications for Models. J. Phys. Chem. A 2006, 110, 12728-12733. 